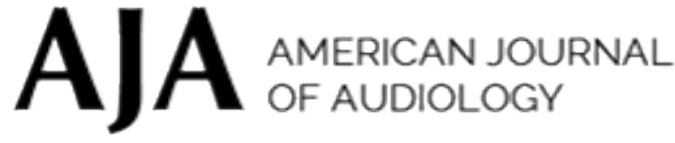

\section{Audiologists and Tinnitus}

\begin{tabular}{|r|l|}
\hline Journal: & The American Journal of Audiology \\
\hline Manuscript ID & Draft \\
\hline Manuscript Type: & Viewpoin \\
\hline Author: & n/a \\
\hline Complete List of Authors: & $\begin{array}{l}\text { Henry, James; VA Medical Center, National Center for Rehabilitative } \\
\text { Auditory Research } \\
\text { Piskosz, Michael; ReSound GN Technology Center } \\
\text { Norena, Arnaud; Aix-Marseille Université } \\
\text { Fournier, Philippe; Universite Aix-Marseille III, Laboratory of Integrative } \\
\text { and Adaptive Neurosciences }\end{array}$ \\
\hline Keywords: & $\begin{array}{l}\text { Assessment, Audiology, Intervention, Tinnitus, Amplification or hearing } \\
\text { aids }\end{array}$ \\
\hline
\end{tabular}

\section{SCHOLARONE ${ }^{m}$ \\ Manuscripts}




\title{
Audiologists and Tinnitus
}

\author{
Authors: James A. Henry, ${ }^{1,2}$ Michael Piskosz, ${ }^{3}$ Arnaud Norena, ${ }^{4}$ Philippe Fournier ${ }^{4}$ \\ ${ }^{1}$ VA RR\&D National Center for Rehabilitative Auditory Research, VA Portland Health Care \\ System, Portland, Oregon \\ ${ }^{2}$ Department of Otolaryngology/Head and Neck Surgery, Oregon Health \& Science University, \\ Portland, Oregon \\ ${ }^{3} \mathrm{GN}$ ReSound, Glenview, Illinois \\ ${ }^{4}$ Laboratory of Integrative and Adaptive Neurosciences, Université Aix-Marseille, Marseille
}

\section{Corresponding author:}

James A. Henry, PhD

VA RR\&D Senior Research Career Scientist

National Center for Rehabilitative Auditory Research

VA Portland Health Care System

3710 SW US Veterans Hospital Rd.

Portland, OR 97239

$503.220 .8262 \times 57466$

Research Professor

Department of Otolaryngology/Head and Neck Surgery

Oregon Health \& Science University, NRC04

3181 SW Sam Jackson Park Rd.

Portland, Oregon, 97201-3098, USA

james.henry@va.gov 


\begin{abstract}
Chronic tinnitus is a complaint of $10-15 \%$ of all adults, and highly prevalent with patients who receive an audiologic assessment. In spite of these numbers, audiologists generally are untrained in best practices for tinnitus management. Audiology graduate programs, as a rule, do not provide comprehensive instruction in tinnitus clinical care. Various training programs exist, but they are inconsistent in their recommendations. Audiologists have the latitude to perform just about any tinnitus procedures they wish as there are no standards to prevent the delivery of unvetted services, which are often expensive for patients. The net result is that patients seeking professional services by an audiologist have no basis upon which to be assured they are receiving evidence-based care. The purpose of this article is to describe the current status of tinnitus management services that exist within the general field of Audiology, and to suggest specific approaches for improving those services.
\end{abstract}




\section{Introduction}

Tinnitus is a "phantom" auditory perception that is experienced chronically by one in 10-15 adults (Bhatt, Lin, \& Bhattacharyya, 2016; Hoffman \& Reed, 2004). For many, the perception disrupts their sleep, interferes with concentration, and causes depression and anxiety (Henry, Dennis, \& Schechter, 2005). These individuals frequently describe high levels of distress and functional limitations. The present authors have worked with thousands of tinnitus sufferers who have described the frustration and hopelessness they come to feel when their healthcare providers can offer them little or no relief. Fortunately, some researchers and clinicians are passionate about, and dedicated to, improving functioning and quality of life for people who experience bothersome tinnitus.

From the manufacturing perspective, tinnitus has a potential market equal to that of hearing aids, considering: (1) $20-40 \%$ of people with hearing loss also have tinnitus (Hoffman \& Reed, 2004). (2) About $80 \%$ of people with tinnitus present some form of hearing loss (Coles, 2000; P. J. Jastreboff \& Hazell, 2004; Nuttall, Meikle, \& Trune, 2004). (3) One study revealed two out of five $(43 \%)$ of people with tinnitus have hearing loss and do not using hearing aids (even though about half of those with both conditions who used hearing aids reported the amplification provided relief from the tinnitus) (Kochkin, Tyler, \& Born, 2011). It can be argued, from a volume perspective, that the device market for tinnitus is potentially even larger than for hearing loss, considering people who might have intermittent tinnitus, or short bouts of tinnitus, which does not generally occur for hearing loss. The market for protective devices for tinnitus patients such as musicians earplugs or more sophisticated plugs is also not negligible.

We are faced with the problem of a high number of people requiring clinical services for tinnitus, but a dearth of healthcare providers who have the knowledge and expertise to provide 
these services competently and expeditiously. Audiologists commonly encounter patients complaining of tinnitus, and audiologists may constitute the primary nexus between these patients and the healthcare community. Because there is no consensus on the management of tinnitus and no cure, otolaryngologists often feel unable to provide effective services and may prefer referring their tinnitus patients to audiologists. Indeed, audiologists may be the bestpositioned healthcare providers to assess tinnitus and to provide intervention if needed, because they:

- $\quad$ are trained and knowledgeable in auditory system functioning and disorders (Searchfield \& Baguley, 2011)

- are skilled in the assessment of hearing function, with these skills being adaptable to evaluate psychoacoustic attributes of tinnitus (Henry, 2004)

- can manage tinnitus patients with sound-based therapies, namely with hearing aids and custom devices that can deliver personalized sound stimulation, which have considerable evidence for alleviating effects of tinnitus (dos Santos et al., 2014; Henry, Frederick, Sell, Griest, \& Abrams, 2015; Henry, McMillan, et al., 2017; Shekhawat, Searchfield, \& Stinear, 2013)

- are generally in possession of good counseling skills, which are needed to be effective tinnitus practitioners (Hall III \& Ruth, 1999; Sweetow, 1985)

- are closely affiliated with otolaryngologists, who are essential to conduct medical evaluations for certain forms of tinnitus (Wackym \& Friedland, 2004)

In spite of all these reasons why audiologists are ideally suited to provide tinnitus services, relatively few of them work with the tinnitus population. Even fewer audiologists provide services according to proper tinnitus management protocols. The purpose of this article is to 
describe the status of tinnitus care within the audiologist community, and to offer recommendations for audiologists to become mutually aligned in their approach to tinnitus management, using evidence from controlled trials, systematic literature reviews, and clinical guidelines based on systematic reviews.

\section{Gaps in Tinnitus Care}

Tinnitus research is prolific_-over 300 tinnitus-related publications appear yearly in peerreviewed journals. With so much research completed, it would seem tinnitus care procedures should be well established and broadly disseminated. In reality, surveys with providers have consistently revealed that tinnitus care is not evidence based, is highly variable across clinics, and does not incorporate standardized measurement of patient outcomes (Carlson et al., 2016; Carlson et al., 2016; Schmidt, Kaelin, Henselman, \& Henry, 2017; Tuepker, Elnitsky, Newell, Zaugg, \& Henry, 2018). Bhatt, Lin, and Bhattacharyya (2016) evaluated the adherence of tinnitus services in the United States to the clinical practice guidelines published by the American Academy of Otolaryngology-Head and Neck Surgery Foundation (AAO-HNSF; Tunkel et al., 2014), and concluded the guidelines were "followed infrequently." This might be explainable because most published trials of tinnitus interventions do not meet the standards of high-quality evidence required by synthesis reviews (e.g., Cochrane). Hence, clinical practice guidelines that rely on such reviews have little basis for making recommendations (Fuller et al., 2017; Tunkel et al., 2014), leaving scant evidence for top-down support from decision and policy makers. It is therefore not surprising that tinnitus assessment and management practices are typically not required curriculum in relevant graduate programs, not only for Audiology but also for Otolaryngology and Mental Health—disciplines that commonly encounter patients complaining of tinnitus. Effective, research-based clinical practices for helping patients manage 
their tinnitus are largely unknown to the great majority of these providers and are therefore not routinely offered in settings where patients seek care.

Many audiologists are overloaded with diagnostics, hearing instrument fittings, and followup visits. They do not have the time to invest into setting up proper tinnitus services in their clinic. Further, there is no clear third-party reimbursement (insurance) for most tinnitus services, resulting in clinicians having to offer these services using a "pay out of pocket" approach, which many are not comfortable requesting (Combs, 2014). Tinnitus evaluation protocols that can provide audiologists with quick, accurate, and convenient results have a better chance of resonating with the clinical community.

Manufacturers and researchers have attempted to educate and train audiologists on tinnitus management services, but with little success judging by the relatively few providers who currently offer such services. For many of the reasons discussed above as to why audiologists do not work with the tinnitus population in spite of their suitability as tinnitus-care providers, it has been difficult to inspire audiologists to ramp up their clinical services to be more inclusive of tinnitus management in their scope of practice. There may not be the payoff for time invested in the minds of many audiologists, in addition to the fact that the large majority are not comfortable in their professional skills with respect to tinnitus. The difficult equation audiologists have to solve is to propose a clinical approach that is efficient in improving the tinnitus condition and at the same time is viable economically. In this context, the "problem" with tinnitus is that it often takes time to manage. No medication is capable of suppressing the percept-management therefore consists of limiting the impact of tinnitus on quality of life. For audiologists, many appointments involving long counseling sessions may be required. What is needed is a standardized and evidence-based clinical management protocol provided at the right cost for both 
patient and provider. It is therefore first an application challenge before being an educational challenge. That being said, there are a few bright shining audiologists who have invested in their training and created tinnitus management services in their clinics, and are profitable as well. These audiologists have committed to learning and implementing tinnitus services into their clinical scope of practice and they have a genuine interest in serving this population. They may typically be the more savvy clinicians and have provided tinnitus services long enough to appreciate the benefit of offering them.

\section{Conducting a Tinnitus Evaluation}

Measuring tinnitus is complicated, considering the only person who can perceive the tinnitus is the patient, thereby making all measurements inherently subjective. In fact, many clinicians and researchers dismiss attempts to measure the psychophysical properties of tinnitus as unhelpful, and choose to focus instead on the patient's reactions to tinnitus (i.e., how much it bothers him or her) (Rabau et al., 2015; Raj-Koziak et al., 2019). Others argue that tinnitus psychoacoustic measures are important for counseling purposes (Basile, Fournier, Hutchins, \& Hebert, 2013; P.J. Jastreboff, 1996; Suzuki, Suzuki, Onishi, \& Penido, 2018). It is not possible to completely escape from measurements of tinnitus perception due to their prevalence in treatment protocols designed to reduce tinnitus loudness, as well as their persistence in medical coding and past literature.

\section{Measuring Tinnitus Perception}

To measure tinnitus perception, four tests (loudness matching, pitch matching, maskability, and residual inhibition) are part of a typical tinnitus psychoacoustic evaluation, which has a CPT code for reimbursement in the United States. The series of tests has been around for over 30 years (Vernon \& Meikle, 1988) and is still performed by some audiologists. Psychoacoustic 
testing for auditory attributes of tinnitus is generally not recommended because the results of testing do not inform clinical decision making, except for pitch matching as described in the next paragraph. A more complete description of these different measures than what is provided here is available elsewhere (Henry, 2016).

Numerous tinnitus sound-therapy methods require a tinnitus pitch match (PM) to shape the sound stimulus that is used with their protocol. As examples: (1) A PM is considered important for Tinnitus Masking to select the optimal masking sound and for monitoring potential changes in the tinnitus percept resulting from treatment (Vernon \& Meikle, 2000). (2) Neuromonics Tinnitus Treatment obtains a PM to shape the broadband noise embedded in their relaxation music (Sinopoli, Davis, \& Hanley, 2007). (3) A method of "notching" the sound stimulus around the PM frequency has been reported effective for reducing the loudness of tinnitus (Okamoto, Stracke, Stoll, \& Pantev, 2010; Pantev, Okamoto, \& Teismann, 2012). (4) The method of Acoustic Coordinated Reset Neuromodulation requires a PM to identify their four stimulation tones - two above and two below the PM frequency (Adamchic et al., 2017). (5) Patients undergoing treatment with the Levo device (Otoharmonics) provide a tinnitus "sound print" to identify the sound most closely matched to the sound of their tinnitus (Theodoroff et al., 2017). Many commercial therapies reliant on knowing the tinnitus pitch use their own method to obtain a PM. Because of the different methods used to obtain a PM, and the generally poor test-retest reliability of pitch matches (Henry, 2016), evidence for the validity of these proprietary soundtherapy methods is equivocal.

Loudness is perhaps the most germane attribute of tinnitus pertaining to treatment intended to reduce the perception of tinnitus. Although not proven, a reduction of tinnitus loudness would logically lessen the impact of tinnitus for an individual (depending of course on the amount of 
loudness reduction and patient characteristics). Tinnitus loudness is typically measured two ways. First, loudness matching is performed such that patients match the loudness of their tinnitus to the loudness of a pure tone-usually at the PM frequency, but also at $1 \mathrm{kHz}$, which may better reflect the actual loudness of tinnitus (Goodwin \& Johnson, 1980). The second method is to use a loudness rating scale, such as $0-10$ (with 10 being the loudest sound imaginable), which is used to select the number that best represents the tinnitus loudness. Loudness rating scales tend to correlate with scores from tinnitus-severity questionnaires (Manning, Grush, Thielman, Roberts, \& Henry, 2019), thus loudness rating scales are not reliable for measuring tinnitus loudness.

Minimum masking level (MML) is not a measure of tinnitus per se, but rather a measure of the susceptibility of tinnitus to being rendered inaudible, or "masked," by an external sound. Testing is usually conducted with broadband noise, and the MML may be different between the two ears. The MML has been reported to be prognostic for patients treated with Tinnitus Masking - those who are easily masked, and find the noise acceptable, are most likely to benefit from masking (Vernon, 1992) MMLs were reportedly reduced for patients following successful sound therapy (Davis, Paki, \& Hanley, 2007; P.J. Jastreboff, Hazell, \& Graham, 1994). Further research is needed to facilitate interpretation of MML measures both for baseline and outcome purposes.

Residual inhibition testing (to determine if sound induces a temporary suppression of tinnitus) is normally conducted after obtaining the MML, by raising the sound to a level above the MML in the attempt to induce the effect. Using the standard clinical test, residual inhibition of brief duration (typically $<1$ minute) was reported to occur for about $90 \%$ of patients (Henry \& Meikle, 2000; Vernon \& Meikle, 2000). The value of testing for residual inhibition is currently 
debated. It has been argued that the testing is not recommended because it can give patients the false sense that tinnitus can be permanently suppressed by sound (P. J. Jastreboff \& Hazell, 2004). Others have argued that residual inhibition can restore hope and a feeling of control (Vernon \& Meikle, 2003). It may very well be there is a way for sound to suppress tinnitus (Fournier et al., 2018), but research has not yet determined how to accomplish that in a systematic and consistent fashion.

\section{Measuring Tinnitus Reactions}

The cure for tinnitus would be to eliminate the phantom sound entirely. This has not yet been discovered, nor has a partial cure (permanent reduction in tinnitus loudness). In lieu of eliminating/reducing the perception of tinnitus, treatment is limited to reducing the functional effects of tinnitus (disruption of sleep, concentration, and/or emotions). At least a dozen questionnaires have been developed and validated for assessing functional effects of tinnitus. Any of these questionnaires is adequate for intake assessment purposes, i.e., to determine the severity of tinnitus and the potential need for intervention. A caveat, however, is that, if a person has both tinnitus and unaddressed hearing loss, that person may ascribe the hearing difficulties to the tinnitus, which would inflate the questionnaire score (Coles, 1995; Ratnayake, Jayarajan, \& Bartlett, 2009; Zaugg, Schechter, Fausti, \& Henry, 2002). When that happens, the tinnitus appears to be a worse problem than it really is. It is therefore argued that administering a tinnitus questionnaire to a person with unaddressed hearing needs can exaggerate the extent of the reported tinnitus problem. The Tinnitus and Hearing Survey (THS) was developed expressly because of this concern (Henry, Griest, et al., 2015). The THS is brief (10 items), and provides a score specific to tinnitus problems and a separate score specific to hearing problems. 
If a patient is to receive intervention for bothersome tinnitus, then it is important to obtain a baseline measure of the tinnitus severity. The same measure is repeated following the intervention to determine if the functional effects of tinnitus have been mitigated. Most tinnitus questionnaires are not validated for tinnitus outcome assessment (i.e., responsiveness). One that is validated for responsiveness is the Tinnitus Functional Index (TFI; Meikle et al., 2012). It is recommended to administer the TFI only after any hearing needs have been met, and prior to intervention. It is then administered following intervention, and the desired result is that the index score is reduced from baseline to post-intervention. In general, perceived benefit from intervention increases in direct proportion to the degree of index score reduction. The TFI includes eight subscales, representing eight different functional domains of tinnitus. Each of these subscales has its own score, so the different domains can be looked at separately to determine within-domain effects of intervention.

The TFI has been translated into over 20 languages. However, not all of these translations have been validated for their psychometric properties. In general, practitioners should use the most validated tinnitus questionnaire for their particular language.

\section{Tinnitus Intervention by Audiologists}

Audiologists have many options available to them to provide intervention for tinnitus. The options are generally limited to using sound in some way to mitigate effects of tinnitus. The commercial methods listed above that require a PM are all possibilities. It needs to be pointed out, however, that no one method of sound therapy has been shown to be any more effective than any other. Further, there are many apps that can be downloaded to smartphones that are either free or very low cost. These free/low cost apps may be as effective as the more expensive sound therapies; they should therefore be the first choice for this purpose. For example, the free 
ReSound Relief app was recently shown to be efficacious for reducing functional effects of tinnitus (Skarzynski, Kutyba, Cywka, \& Sztabnicka, 2019). If tinnitus apps have been tried for a patient without success, then the more expensive devices can be considered-assuming they are desired and affordable by the patient.

It should be further pointed out that "sound therapy" is not recommended by the AAO-HNSF guidelines (Tunkel et al., 2014). Rather, it is considered "optional” for tinnitus intervention because of the relative lack of randomized controlled trials (RCTs) demonstrating the efficacy of different methods of sound therapy. Sound therapy does not have as strong an evidence-base as for Cognitive Behavioral Therapy (CBT), but that may be because of the limited RCTs for sound therapy reported in the literature — not necessarily because sound therapy is any less effective. It is suggested, however, that patients with very bothersome tinnitus be referred to a behavioral health provider who has expertise in CBT for tinnitus. Such providers may be difficult to find, but many have expertise in CBT for depression and anxiety. It would seem likely that CBT in general would be helpful to teach patients coping skills for tinnitus management.

The method of Progressive Tinnitus Management (PTM) uses a stepped-care approach. Patients receive an audiologic exam and hearing aids if warranted (Henry \& Manning, 2019) If their tinnitus is bothersome following the audiologic services, a combination of sound therapy and $\mathrm{CBT}$ is recommended for intervention. The intervention involves teaching sessions that focus on using sound as therapy, and certain skills derived from CBT (relaxation, distraction activities, and changing thoughts about tinnitus) (Henry, Zaugg, Myers, \& Kendall, 2010). PTM has been vetted for efficacy in a multi-site RCT (Henry, Thielman, et al., 2017).

Although PTM is mentioned, other methods of tinnitus management have been developed that have various levels of research evidence. It will be important to conduct RCTs to evaluate 
each of these therapies by comparing effect sizes and the duration of treatment required to obtain each effect size.

\section{Suggested Protocol for Audiologists}

As already argued, audiologists are the clinicians in the best position to at least initiate tinnitus clinical services. Audiologists can be considered the gateway to healthcare services for tinnitus sufferers. Those who currently provide tinnitus services often just do what they think is best (or most profitable) without following research-based guidelines. A research-based protocol for audiologists that requires very little additional time ( $\sim 5$ minutes) to determine what a patient's needs are with respect to tinnitus and hyperacusis is available (Henry \& Manning, 2019) Audiologists need to at least know what to do when a patient reports the presence of tinnitus, and this brief protocol will address that concern. The protocol involves essentially administration of the THS along with a standard audiologic evaluation. The information derived from that testing would normally be sufficient to know if the patient requires intervention for tinnitus, for hearing loss, or for both. The THS also provides screening for a sound tolerance problem (hyperacusis/misophonia), which can also be largely addressed by audiologists (Henry et al., 2010).

Approximately $80 \%$ of all people with tinnitus (not just those looking for help with tinnitus) have hearing loss (Kim et al., 2011). There is considerable evidence that providing amplification through hearing aids for people with hearing loss can be beneficial in reducing functional effects of tinnitus (dos Santos et al., 2014; Henry, Frederick, et al., 2015; Henry, McMillan, et al., 2017; Shekhawat et al., 2013). Although more controlled research is needed, an abundance of evidence supports the use of hearing aids for tinnitus management. So, if "profitability" is a concern for audiologists, the fact that hearing aids or combination instruments have research evidence for 
tinnitus management, along with a 5-minute protocol for conducting the tinnitus assessment with the THS, should be sufficient to convince many audiologists to add tinnitus services to their practice.

\section{Conclusions}

Because of the diverse and ethereal nature of tinnitus, it is important to develop a global/holistic view of the field of clinical management for this condition. The field is composed primarily of four players: patients, clinicians, researchers, and industry — who should interact strongly with each other. Researchers and clinicians should listen to patients and understand their concerns and needs. Patient needs are diverse and the corresponding clinical management should be adapted and individualized. Researchers need to develop the appropriate tests to ideally identify tinnitus sub-groups and orient patients to the adapted treatment. Clinicians can of course add to our knowledge base because of their direct interaction with patients on a daily basis.

Whereas this global/holistic view is the ideal, we are far from achieving that goal. In the meantime, audiologists can augment their basic skills to become a cadre of competent tinnitus specialists. The challenge is to provide clinical services that are consistent, research based, and in the patients' best interest with respect to cost of services and timeliness. Any audiologist can perform a basic tinnitus assessment with the THS and provide hearing aids or combination instruments as intervention for tinnitus. As explained above, these basic tinnitus services require very little time on top of standard audiologic services. A higher level of services depends on whether the services are viable economically. These higher-level services should find the right balance between efficacy and time and cost for patients. Follow-up is a sine qua non to provide adequate care for these patients. Some audiologists are interested in advanced training, which elevates them to the status of providing more extensive tinnitus services. If the majority of 
audiologists would learn to provide basic tinnitus services, then only a subgroup would need to become proficient in providing more comprehensive tinnitus services. This scenario is achievable if audiologists will coordinate their efforts with this goal in mind.

\section{Acknowledgments}

Innumerable individuals have contributed indirectly to this manuscript. The authors wish to specifically acknowledge Candice Manning, PhD, AuD, Tara Zaugg, AuD, Christine Kaelin, MBA, and Emily Thielman, MS for their significant contributions, all of whom are employed at the VA RR\&D National Center for Rehabilitative Auditory Research.

\section{Conflict of Interest Statement}

Dr. Henry is funded by the Department of Veterans Affairs, Rehabilitation Research \& Development (RR\&D) Service: Senior Research Career Scientist Award (1 IK6 RX002990-01). Mr. Piskosz is employed by GN ReSound Corp. Drs. Fournier and Norena are employed at the Laboratory of Integrative and Adaptive Neurosciences, Université Aix-Marseille, Marseille. None of the authors has a conflict of interest, financial or otherwise, with the content of this article.

\section{References}

Adamchic, I., Toth, T., Hauptmann, C., Walger, M., Langguth, B., Klingmann, I., \& Tass, P. A. (2017). Acute effects and after-effects of acoustic coordinated reset neuromodulation in patients with chronic subjective tinnitus. Neuroimage Clin, 15, 541-558. doi:10.1016/j.nicl.2017.05.017

Basile, C. E., Fournier, P., Hutchins, S., \& Hebert, S. (2013). Psychoacoustic assessment to improve tinnitus diagnosis. PLoS One, 8(12), e82995.

Bhatt, J. M., Lin, H. W., \& Bhattacharyya, N. (2016). Prevalence, Severity, Exposures, and Treatment Patterns of Tinnitus in the United States. JAMA Otolaryngol Head Neck Surg, 142(10), 959-965.

Carlson, K., Thielman, E., Zaugg, T., Elnitsky, C., Tuepker, A., Kaelin, C., \& Henry, J. A. (2016). Factors affecting the provision of evidence-based Progressive Tinnitus Management in Department of Veterans Affairs medical centers. Paper presented at the Academy Health Annual Research Meeting, Boston, MA. 
Carlson, K. C., Thielman, E., Zaugg, T. L., Elnitsky, C., Tuepker, A., Kaelin, C., \& Henry, J. A. (2016). VA Clinician Surveys and Interviews Reveal Need for Increased Mental Health Involvement in Tinnitus Management. Paper presented at the Joint Defense Veterans Audiology Conference (JDVAC), St Louis, MO.

Coles, R. R. A. (1995). Classification of causes, mechanisms of patient disturbance, and associated counseling. In J. A. Vernon \& A. R. Moller (Eds.), Mechanisms of Tinnitus (pp. 11-19). Needham Heights: Allyn \& Bacon.

Coles, R. R. A. (2000). Medicolegal issues. In R. S. Tyler (Ed.), Tinnitus Handbook (pp. 399417). San Diego: Singular.

Combs, L. M. (2014). Tinnitus Management in Private Practice. (AuD thesis), Ohio State University.

Davis, P. B., Paki, B., \& Hanley, P. J. (2007). Neuromonics tinnitus treatment: Third clinical trial. Ear and Hearing, 28(2), 242-259.

dos Santos, G. M., Bento, R. F., de Medeiros, I. R., Oiticcica, J., da Silva, E. C., \& Penteado, S. (2014). The influence of sound generator associated with conventional amplification for tinnitus control: randomized blind clinical trial. Trends Hear, 18.

Fournier, P., Cuvillier, A. F., Gallego, S., Paolino, F., Paolino, M., Quemar, A., . . Norena, A. (2018). A New Method for Assessing Masking and Residual Inhibition of Tinnitus. Trends Hear, 22.

Fuller, T. E., Haider, H. F., Kikidis, D., Lapira, A., Mazurek, B., Norena, A., . . Cima, R. F. (2017). Different Teams, Same Conclusions? A Systematic Review of Existing Clinical Guidelines for the Assessment and Treatment of Tinnitus in Adults. Front Psychol, 8, 206.

Goodwin, P. E., \& Johnson, R. M. (1980). The loudness of tinnitus. Acta Otolaryngologica, 90, $353-359$.

Hall III, J. W., \& Ruth, R. A. (1999). Outcome for tinnitus patients after consultation with an audiologist. In J. Hazell (Ed.), Proceedings of the Sixth International Tinnitus Seminar (pp. 378-380). London: The Tinnitus and Hyperacusis Centre.

Henry, J. A. (2004). Audiologic assessment of the tinnitus patient. In J. B. Snow (Ed.), Tinnitus: Theory and Management (pp. 220-236). Lewiston, NY: BC Decker, Inc.

Henry, J. A. (2016). "Measurement" of Tinnitus. Otol Neurotol, 37(8), e276-285.

Henry, J. A., Dennis, K., \& Schechter, M. A. (2005). General review of tinnitus: Prevalence, mechanisms, effects, and management. Journal of Speech, Language, and Hearing Research, 48(5), 1204-1234.

Henry, J. A., Frederick, M., Sell, S., Griest, S., \& Abrams, H. (2015). Validation of a novel combination hearing aid and tinnitus therapy device. Ear Hear, 36(1), 42-52.

Henry, J. A., Griest, S., Zaugg, T. L., Thielman, E., Kaelin, C., Galvez, G., \& Carlson, K. F. (2015). Tinnitus and hearing survey: a screening tool to differentiate bothersome tinnitus from hearing difficulties. Am J Audiol, 24(1), 66-77.

Henry, J. A., \& Manning, C. (2019). Clinical protocol to promote standardization of basic tinnitus services by audiologists. Am J Audiol 28(1S):152-161.

Henry, J. A., McMillan, G., Dann, S., Bennett, K., Griest, S., Theodoroff, S., . . Saunders, G. (2017). Tinnitus Management: Randomized Controlled Trial Comparing Extended-Wear Hearing Aids, Conventional Hearing Aids, and Combination Instruments. J Am Acad Audiol, 28(6), 546-561.

Henry, J. A., \& Meikle, M. B. (2000). Psychoacoustic measures of tinnitus. Journal of the American Academy of Audiology, 11, 138-155. 
Henry, J. A., Thielman, E. J., Zaugg, T. L., Kaelin, C., Schmidt, C. J., Griest, S., . . Carlson, K. (2017). Randomized Controlled Trial in Clinical Settings to Evaluate Effectiveness of Coping Skills Education Used With Progressive Tinnitus Management. Journal of speech, language, and hearing research: JSLHR, 60(5), 1378-1397.

Henry, J. A., Zaugg, T. L., Myers, P. M., \& Kendall, C. J. (2010). Progressive Tinnitus Management: Clinical Handbook for Audiologists. San Diego, CA: Plural Publishing.

Hoffman, H. J., \& Reed, G. W. (2004). Epidemiology of tinnitus. In J. B. Snow (Ed.), Tinnitus: Theory and Management (pp. 16-41). Lewiston, NY: BC Decker Inc.

Jastreboff, P. J. (1996). Usefulness of the psychoacoustical characterization of tinnitus. In G. E. Reich \& J. A. Vernon (Eds.), Proceedings of the Fifth International Tinnitus Seminar 1995 (pp. 158-166). Portland: American Tinnitus Association.

Jastreboff, P. J., \& Hazell, J. W. P. (2004). Tinnitus Retraining Therapy: Implementing the Neurophysiological Model. New York: Cambridge University Press.

Jastreboff, P. J., Hazell, J. W. P., \& Graham, R. L. (1994). Neurophysiological model of tinnitus: dependence of the minimal masking level on treatment outcome. Hearing Research, 80, 216232.

Kim, D. K., Park, S. N., Kim, H. M., Son, H. R., Kim, N. G., Park, K. H., \& Yeo, S. W. (2011). Prevalence and significance of high-frequency hearing loss in subjectively normal-hearing patients with tinnitus. Ann Otol Rhinol Laryngol, 120(8), 523-528.

Kochkin, S., Tyler, R., \& Born, J. (2011). MarkeTrak VIII: The prevalence of tinnitus in the United States and the self-reported efficacy of various treatments. Hearing Review, 18(12), 10-26.

Manning, C., Grush, L., Thielman, E., Roberts, L., \& Henry, J. A. (2019). Comparison of Tinnitus Loudness Measures: Matching, Rating, and Scaling. Am J Audiol, 28(1), 137-143.

Meikle, M. B., Henry, J. A., Griest, S. E., Stewart, B. J., Abrams, H. B., McArdle, R., . . . Vernon, J. A. (2012). The tinnitus functional index: development of a new clinical measure for chronic, intrusive tinnitus. Ear Hear, 33(2), 153-176.

Nuttall, A. L., Meikle, M. B., \& Trune, D. R. (2004). Peripheral processes involved in tinnitus. In J. B. Snow (Ed.), Tinnitus: Theory and Management (pp. 52-68). Lewiston, NY: BC Decker Inc.

Okamoto, H., Stracke, H., Stoll, W., \& Pantev, C. (2010). Listening to tailor-made notched music reduces tinnitus loudness and tinnitus-related auditory cortex activity. Proc Natl Acad Sci U S A, 107(3), 1207-1210.

Pantev, C., Okamoto, H., \& Teismann, H. (2012). Tinnitus: the dark side of the auditory cortex plasticity. Ann N Y Acad Sci, 1252(1), 253-258.

Rabau, S., Cox, T., Punte, A. K., Waelkens, B., Gilles, A., Wouters, K., . . V Van de Heyning, P. (2015). Changes over time of psychoacoustic outcome measurements are not a substitute for subjective outcome measurements in acute tinnitus. Eur Arch Otorhinolaryngol, 272(3), 573581.

Raj-Koziak, D., Gos, E., Swierniak, W., Karpiesz, L., Niedzialek, I., Wlodarczyk, E., . . . Skarzynski, P. H. (2019). Relationship Between Tinnitus Loudness Measure by Visual Analogue Scale and Psychoacoustic Matching of Tinnitus Loudness. Otol Neurotol, 40(1), 16-21.

Ratnayake, S. A., Jayarajan, V., \& Bartlett, J. (2009). Could an underlying hearing loss be a significant factor in the handicap caused by tinnitus? Noise Health, 11(44), 156-160. 
Schmidt, C. J., Kaelin, C., Henselman, L., \& Henry, J. A. (2017). Need for mental health providers in Progressive Tinnitus Management: A gap in clinical care. Federal Practitioner, 34(5), 6-9.

Searchfield, G. D., \& Baguley, D. M. (2011). The role of the audiologist in tinnitus practice. In A. R. Moller, B. Langguth, D. DeRidder, \& T. Kleinjung (Eds.), Textbook of tinnitus (pp. 215-222). New York, NY: Springer.

Shekhawat, G. S., Searchfield, G. D., \& Stinear, C. M. (2013). Role of hearing AIDS in tinnitus intervention: a scoping review. J Am Acad Audiol, 24(8), 747-762. doi:10.3766/jaaa.24.8.11

Sinopoli, T., Davis, P. B., \& Hanley, P. (2007). Tinnitus: addressing neurological, audiological, and psychological aspects with customized therapy. Hearing Review, 14(9), 32-35.

Skarzynski, P. H., Kutyba, J., Cywka, K., \& Sztabnicka, A. (2019). Effects of Using the ReSound Relief Application in Therapy of Tinnitus. Paper presented at the American Academy of Audiology Annual Convention, Nashville, TN.

Suzuki, F. A. B., Suzuki, F. A., Onishi, E. T., \& Penido, N. O. (2018). Psychoacoustic classification of persistent tinnitus. Braz J Otorhinolaryngol, 84(5), 583-590.

Sweetow, R. (1985). Counseling the patient with tinnitus. Arch Otolaryngol, 111(5), 283-284.

Theodoroff, S. M., McMillan, G. P., Zaugg, T. L., Cheslock, M., Roberts, C., \& Henry, J. A. (2017). Randomized controlled trial of a novel device for tinnitus sound therapy during sleep. Am J Audiol, 1-12.

Tuepker, A., Elnitsky, C., Newell, S., Zaugg, T., \& Henry, J. A. (2018). A qualitative study of implementation and adaptations to Progressive Tinnitus Management (PTM) delivery. PLoS One, 13(5), e0196105.

Tunkel, D. E., Bauer, C. A., Sun, G. H., Rosenfeld, R. M., Chandrasekhar, S. S., Cunningham, E. R., Jr., . . . Whamond, E. J. (2014). Clinical practice guideline: tinnitus. Otolaryngol Head Neck Surg, 151(2 Suppl), S1-S40.

Vernon, J. A. (1992). Tinnitus: causes, evaluation, and treatment. In G. M. English (Ed.), Otolaryngology (Revised Edition) (pp. 1-25). Philadelphia: JB Lippincott.

Vernon, J. A., \& Meikle, M. B. (1988). Measurement of tinnitus: an update. In M. Kitahara (Ed.), Tinnitus. Pathophysiology and Management (pp. 36-52). Tokyo: Igaku-Shoin.

Vernon, J. A., \& Meikle, M. B. (2000). Tinnitus masking. In R. S. Tyler (Ed.), Tinnitus Handbook (pp. 313-356). San Diego: Singular Publishing Group.

Vernon, J. A., \& Meikle, M. B. (2003). Tinnitus: clinical measurement. The Otolaryngologic Clinics of North America, 36(2), 293-305, vi.

Wackym, P. A., \& Friedland, D. R. (2004). Otologic evaluation. In J. B. Snow (Ed.), Tinnitus: Theory and Management (pp. 205-219). Lewiston, NY: BC Decker Inc.

Zaugg, T. L., Schechter, M. A., Fausti, S. A., \& Henry, J. A. (2002). Difficulties caused by patients' misconceptions that hearing problems are due to tinnitus. In R. Patuzzi (Ed.), Proceedings of the Seventh International Tinnitus Seminar (pp. 226-228). Crawley: The University of Western Australia. 